\title{
Productividad y sostenibilidad del cultivo de sorgo forrajero como alternativa para la alimentación de rumiantes
}

\author{
Productivity and sustainability of sorgum forage crop as an \\ alternative for feeding ruminants
}

\author{
Diana Katherinne Rios Moyano* \\ Universidad La Salle Colombia (Colombia) \\ Abelardo Conde Pulgarín \\ Universidad La Salle Colombia (Colombia) \\ Claudia Fernanda Rios Moyano \\ Universidad Pedagógica y Tecnológica de Colombia (Colombia)
}

Recibido: 15 de octubre de 2020

Aceptado: 26 de mayo de 2021

Publicado: 10 de noviembre de 2021

\section{Resumen}

El sorgo (Sorghum vulgare) es un cultivo utilizado como alternativa de alimentación bovina, por medio de la conservación en silo, siendo altamente funcional con respecto a metodologías tradicionales como el pastoreo de forraje nativo o mejorado. Es un cultivo con buena adaptación, tolerancia a condiciones de sequía, elevada producción de materia verde y capacidad de rebrote de las plantas gracias a su rápido crecimiento. Por tanto, el objetivo del presente trabajo es analizar la productividad y sostenibilidad del cultivo de sorgo para la alimentación bovina, con especial énfasis en la zona de vida bosque húmedo tropical bh-T, como suplemento forrajero; por medio de metodologias de análisis-síntesis de la sostenibilidad en las dimensiones ambiental, social, económica, institucional y técnica, además mostrar los resultados de evaluación productiva de siete genotipos de Sorgo como base forrajera para la elaboración de silo y así para brindar una herramienta al momento de implementar estrategias de alimentación aplicadas a los sistemas de producción bovina. Se destaca a través del análisis realizado para el cultivo de sorgo "Email: dkrios@unisalle.edu.co

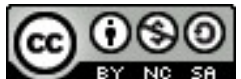

Revista del Centro de Investigación. Universidad La Salle por Dirección de Investigación. Universidad La Salle Ciudad de México se distribuye bajo una Licencia Creative Commons Atribución-NoComercial-CompartirIgual 
Rios Moyano, D. K.; Conde Pulgarín, A.; Rios Moyano, C. F.

que, en los indicadores de sostenibilidad propuestos, el silo de sorgo es medianamente sostenible con un promedio en las cinco dimensiones evaluadas de $73 \%$ de cumplimiento, donde el mayor porcentaje de cumplimiento corresponde a las dimensiones medio ambiental y técnica (76\%). Igualmente, se identificaron variedades de sorgo con niveles de producción adecuados para el ecosistema del departamento de Casanare, donde el genotipo ABS 7000, obtuvo el segundo puesto en producción de biomasa en fresco de 66,95 T/ha y una proporción de materia seca intermedia (MS) 18,72\%.

Palabras clave: Alimentación bovina; sostenibilidad; bancos forrajeros; ganadería; productividad 


\section{Abstract}

Sorghum (Sorghum vulgare) is a crop that has been visualized as another bovine feeding alternative, preserved by silo for cattle, because it is highly functional with respect to traditional feeding methodologies such as native or improved forage grazing. It is important to note that sorghum is considered a crop with good adaptation and tolerance to drought conditions, which are limiting for other types of forage; it also has a high production of green matter and an efficient regrowth capacity of plants since it is a fast growing species. According to the above, the objective of this work is to analyze and evaluate the sorghum-based livestock feed bank model as a sustainable alternative as a supplement for bovine nutrition in the life zone Tropical moist forest, through analysis-synthesis of sustainability of the sorghum silo in the environmental, social, economic, institutional and technical areas, and showing results of productive evaluation of seven Sorgo genotypes, as a forage base for silo production, providing a tool to implement feeding strategies applied to bovine production systems. Through the analysis went for the cultivation of sorghum, in the proposed sustainability indicators, the sorghum silo is moderately sustainable with an average in the five dimensions evaluated of $73 \%$ compliance, where the highest percentage of compliance corresponds to the environmental and technical dimensions (76\%). Besides, sorghum varieties were identified with adequate production levels for the ecosystem of the department of Casanare, where the ABS 7000 was in second place in fresh biomass of $66.95 \mathrm{~T} /$ ha and a proportion of intermediate dry matter (DM) $18.72 \%$.

Keywords: Bovine feeding; sustainability; fodder banks; cattle raising; productivity 
Rios Moyano, D. K.; Conde Pulgarín, A.; Rios Moyano, C. F.

\section{Introducción}

El consumo de alimentos mundialmente ha evolucionado y está en constante cambio, muchas de las actividades agropecuarias utilizadas actualmente han generado impactos en la salud y bienestar humano, asi como en los ecosistemas; las cadenas productivas de alimentos de origen animal como la actividad ganadera reportan efectos negativos a nivel ambiental, tales como degradación de suelos, subutilizacion o uso ineficiente de resursos (FAO, 2016; Mora et al., 2017). Los cambios ambientales mundiales son una amenaza constante para la seguridad alimentaria y nutriciónal de la población y las generaciones futuras; por lo que es urgente la adopción de vías específicas que faciliten la transición hacia sistemas agrícolas y alimentarios más sostenibles; sumando a lo anterior, los escasos recursos naturales y el aumento en la demanda de alimentos, en particular de origen animal, puede generar oportunidades de innovación para el desarrollo agrícola, incluida la ganadería, que sean sustentables ambientalemente (FAO, 2016).

Es así como, el sector ganadero ha sufrido una transformación importante en las últimas décadas; la creciente demanda de alimentos derivados de los animales en las economías que más rápido crecen en el mundo ha incrementado significativamente la producción ganadera, con la ayuda de importantes innovaciones tecnológicas y cambios estructurales en el sector. Esta creciente demanda, se ha satisfecho principalmente por la producción ganadera comercial y las cadenas alimentarias asociadas. Mundialmente la ganadería tiene cerca del $80 \%$ de uso de las tierras agrícolas destinadas a la producción de forrajes y alimento para el ganado, contribuyendo con cerca de un $40 \%$ de la producción en paises desarrollados y $20 \%$ en paises en desarrollo; aportando así al sostenimiento de cerca de 1.300 millones de personas a nivel global (Gutiérrez et al., 2018; FAO, 2020a). En los sistemas de producción bovina para asegurar y mejorar la eficiencia productiva es esencial mantener la alimentación constante y disponible para los animales con el fin de disminuir la variabilidad en la producción de leche y carne; la conservación de alimento estabiliza la carga animal en las fincas, ya que permite el uso de este en épocas de escasez (Villegas y Trujillo, 2014). Uno de los sectores agrícolas mas relacionado con la ganadería es el de los cereales, ya que estos representan cerca del 13\% de ingesta de los bovinos, proyectándose un aumento del consumo de cereales y oleaginosas en la próxima década debido a la necesidad de forrajes aptos para consumo animal, esto se relaciona de forma directa con la necesidad de alimentos de origen animal para el ser humano (FIRA, 2019; FAO, 2020a).

Con respecto a los modelos actuales de alimentación del ganado, se puede señalar que los países desarrollados utilizan dos tipos de alimentación para el ganado, la alimentación de pasto y el ensilaje, consideradas como las mejores alternativas para la obtención de carnes de calidad, al tiempo que reducen los efectos negativos sobre el medio ambiente ya que la biomasa de la planta es totalmente consumida por el ganado y no es necesario realizar la quema de residuos de cosecha; además, el estiércol de rumiantes se utiliza como fertilizante en el campo para mantener una práctica agrícola más verde y sostenible (Xie y Xu, 2019).

Según la FAO (2020b) "el crecimiento acelerado de la ganadería ha convertido a América Latina en el mayor exportador de carne bovina, lo que representa alrededor del 45\% del PIB agrícola de la región". En América tropical el mayor uso de la tierra de los agroecosistemas se encuentra en pasturas, llegando en algunos países a ocupar entre el 60-80\% del área (Murgueitio y Ibrahim, 2004). En cuanto al modelo 
de alimentación bovina latinoamericano, se habla de que en la mayoría de los casos ha primado la expansión de las áreas dedicadas a pasturas (Pezo, 2019) Para el caso de los sistemas de ganadería colombiana, esta se basan en alimento a base de pasturas, usando la suplementación casi siempre en épocas críticas por escasez de comida como sucede en épocas de verano, sin embargo no se cuenta con una cultura de ensilar (Santos, 2015), lo anterior conlleva a que los parámetros productivos sean bajos en la mayoría de las regiones ganaderas, limitando la competitividad productiva ya que la baja oferta forrajera se ve limitada por las condiciones agroecológicas propias de las regiones tales como, regímenes de lluvias o periodos de sequía, generando impactos negativos en las pasturas y praderas, sumado a esto existen limitantes técnicas, tecnológicas, de infraestructura y sanitarias que no permiten alcanzar una sostenibilidad y competitividad de los sistemas productivos (Mahecha, Gallego y Peláez, 2002; FEDEGAN, 2013)

Así mismo, en el departamento de Casanare, Colombia; la ganadería se caracteriza por contar con amplias explotaciones de bovinos en zonas de llanura, zona con características edafo-climáticas que dificultan contar con alimento disponible para los animales de forma constante, dentro de las que se destacan largos periodos de sequia, acidez de suelos y bajos niveles de fertilidad. En las regiones con clima cálido las praderas presentan problemas de deficiencia proteica y digestibilidad baja; una alternativa viable que aporte en la solución de esta problemática es el establecimiento de «bancos de proteína», compuestos principalmente por leguminosas (SENA, 2009).

Por lo tanto, la sostenibilidad de la alimentación bovina, se convierte en una necesidad continua por lo que deben diseñarse alternativas que incluyan el uso de bancos forrajeros elaborados con cultivos de ciclo corto que posean ventajas agrícolas y nutricionales para las explotaciones bovinas. Una opción es la elaboración de silos, ya que estos se pueden mantener por un año conservando la calidad nutricional del forraje de forma estable a través del tiempo, evitando pérdidas económicas ya que se mantiene una producción sostenida de alimento durante todo el año, facilitando la intensificación de la producción. Los cultivos más utilizados para el ensilaje a nivel mundial son el maíz, alfalfa, sorgo y pastos, aunque también se ensilan trigo, sorgo y algunas legumbres (Garcés Molina et al., 2004; FAO, 2005; Santos, 2015). Es importante recalcar, que en cuanto a las fuentes de elaboración de bancos de alimentación ganadera, el sorgo es uno de los cultivos que se destaca como fuente de alimento económico y sostenible (Morell, Exposito y Ruiz, 2018; Xie y Xu, 2019).

En el caso particular del sorgo en Colombia, en 2017 se sembraron 12.930 hectáreas de la semilla en el país, con una producción promedio de 50 toneladas de ensilaje por hectárea al año (Ministerio de Agricultura y Desarrollo Rural - MinAgricultura, 2018). Por lo tanto, este trabajo analiza el potencial productivo de siete genotipos de sorgo de origen nacional e internacional y los factores que determinan su sostenibilidad, como alternativa para la alimentación de en especial ganado bovino en ecosistemas de bosque húmedo tropical, generando información esencial para la toma de decisiones del ganadero al momento de implementar estrategias de alimentación aplicadas a los sistemas de producción bovina. 
Rios Moyano, D. K.; Conde Pulgarín, A.; Rios Moyano, C. F.

\section{Materiales y Métodos}

Este trabajo se realizó en el departamento de Casanare (Colombia) teniendo en cuenta que su economía se basa principalmente en la producción agrícola y ganadera (Rojas, 2020) y que las condiciones agroclimáticas del municipio de Yopal son acordes a las necesidades del cultivo de Sorgo.

Dentro de la metodología de este trabajo se identificaron dos etapas generales I Etapa. Evaluación de Sostenibilidad del cultivo de Sorgo y II Etapa. Evaluación de la productividad de genotipos de sorgo. La descripción detallada de cada una de las etapas se muestra a continuación.

\section{Etapa. Evaluación de Sostenibilidad del cultivo de Sorgo}

Para el desarrollo de esta etapa se siguieron varios procesos utilizando métodos teóricos y empíricos destacando el análisis-síntesis, revisión de documentos, entrevista semiestructurada y observación. La utilidad y uso de este procedimiento metodológico se basa en la detección de puntos críticos de la sostenibilidad, establecer sus causas y proponer soluciones a mediano plazo. Así, al tratar el desarrollo sostenible con un enfoque integrado, se utiliza un marco conceptual integrado de indicadores que consideren las cuatro dimensiones básicas de la sostenibilidad: socio-cultural, económico, medioambiental, político-institucional; el uso de una metodología y de indicadores permite observar claras tendencias en el desarrollo de los sistemas productivos (Bolívar, 2011). El sorgo al plantearse como alternativa para la elaboración de silo, requiere evaluarse bajo un esquema de sostenibilidad adaptado para los sistemas de producción agrícolas. La descripción de los procesos de esta etapa se detalla a continuación:

a) Determinación de los puntos críticos. Para este aspecto se tomaron como base tres entrevistas realizadas en el 2018 a administradores de sistemas de producción bovina ubicados en la ciudad de Yopal, Casanare, los cuales contaban con sistemas de producción de silo (sorgo y maíz), a través de estas entrevistas se identificaron fortalezas y debilidades del sistema de silo, las cuales fueron expuestas en una matriz FODA.

b) Selección de indicadores. La evaluación de la sostenibilidad del sistema productivo agrícola de sorgo, se relacionó con la determinación de los criterios de diagnóstico y selección de indicadores estratégicos. Para ello se utilizó la metodología de valoración de indicadores de las dimensiones ambientales, económicas, sociales más el aporte institucional y técnico de acuerdo a la Guía Metodológica de Diseño de Indicadores Compuestos de Desarrollo Sostenible propuesta por Cepal, 2009 (Schuschny y Soto, 2009).

Los indicadores se definieron mediante un proceso de análisis para cada parámetro a evaluar, teniendo en cuenta las cinco dimensiones propuestas para este trabajo; además se utilizó la escala de Likert de $1-5$ para su calificación (Schuschny y Soto, 2009). Cada dimensión se analizó por separado mediante la sumatoria de las calificaciones obtenidas para sus elementos y así se obtuvo una puntuación total, que se comparó con una calificación "ideal”" establecida para cada elemento generando el porcentaje total que se ubicó dentro de una nueva escala definiendo: Enfoque sostenible alto (80\% - 100\%), medio (50\% - 79\%) y bajo $(0 \%-49 \%)$ para el este estudio. 
c) Análisis de las dimensiones de sostenibilidad del sistema: Las evaluaciones en estas dimensiones se realizaron a través de criterios diagnósticos que permitieron construir indicadores del estado del sistema; analizando de esta forma las dimensiones sociales, ambiental, económica, institucional y técnica, tales dimensiones se describen en la tabla 1.

Tabla 1.

Descripción de las dimensiones incluidas en el modelo de sostenibilidad

\section{Dimensión $\quad$ Descripción de la dimensión}

Social

Se orienta a una mejor calidad de vida (superar la pobreza, satisfacer las necesidades básicas humanas e igualar los ingresos), reasignando los recursos económicos para atender estas necesidades. Asimismo es de máxima importancia lograr la estabilidad demográfica, detener el sobreconsumo, y avanzar hacia la formación del capital humano y social.

Medioambiental Considera aquellos aspectos que tienen que ver con preservar y potenciar la diversidad y complejidad de los ecosistemas, su productividad, los ciclos naturales y la biodiversidad. La utilización de los recursos naturales y energéticos se limita a la capacidad de regeneración de éstos y la generación de los residuos a la capacidad de asimilación del ecosistema

Económica Incluye a todo el conjunto de actividades humanas relacionadas con la producción, distribución y consumo de bienes y servicios, es decir, qué tan eficiente es la combinación de los recursos tierra, trabajo y capital. Incluye, por lo tanto, todas las actividades relacionadas a la producción, costos, ingresos, beneficios, entre otros, de las empresas agrícolas a medio y largo plazo.

Institucional Cobra particular interés en el proceso de democratización y participación ciudadana. El principio que la sustenta es que la democracia viabilidad la reorientación del camino del desarrollo y, por lo tanto, la reasignación de recursos hacia diferentes actividades y grupos sociales. Implica realizar progresos significativos en la descentralización política administrativa de las decisiones, para estimular nuevas formas de organización y participación ciudadana.

Técnica Se requiere una aceleración de la innovación y el desarrollo tecnológicos para reducir el contenido en recursos naturales de determinadas actividades económicas, así como para mejorar la calidad de la producción. Implica la búsqueda y cambio hacia tecnologías más eficientes en el caso de los países industrializados y el desarrollo de tecnologías más eficientes y limpias en países en vías de rápida industrialización. En los países en desarrollo con economías basadas en la agricultura, es necesario desarrollar tecnologías apropiadas y de pequeña escala para el incremento de la productividad agrícola.

Fuente: (Bolívar, 2011). Adaptado de nuestra esfera Cinco dimensiones para avanzar hacia un desarrollo sustentable, disponible en http://nuestraesfera.cl/zoom/cinco-dimensiones-para-avanzar-hacia-un-desarrollo-sustentable/ 
Rios Moyano, D. K.; Conde Pulgarín, A.; Rios Moyano, C. F.

\section{Etapa. Evaluación de la productividad de genotipos de sorgo}

Para esta etapa se trabajó un análisis basado en la investigación de adaptación de genotipos de sorgo realizada en condiciones del ecosistema de Bosque húmedo tropical (bh- $\mathrm{T}$ ) en el segundo semetre de 2016 (2016B) en La finca Matepantano de la Universidad de la Salle, ubicada en Yopal Casanare, en el Piedemonte casañero en el centro nororiental de Colombia, en la región de la Orinoquía; a una altura de 256 m s. n. m. y latitud norte $5^{\circ} 19^{\prime} 50^{\prime \prime}$ y latitud oeste de $72^{\circ} 23^{\prime} 26^{\prime \prime}$; con una temperatura promedio anual de $24^{\circ} \mathrm{C}$ y precipitación anual de 2.000 a $4.000 \mathrm{~mm}$.

Se sembraron siete geotipos de sorgo de origen Nacional e internacional, disponibles comercialmente en el país que se caracterizan por presentar una oferta doble propósito, pastoreo directo y para la elaboración de silo (ver tabla 2). Para la siembra se seleccionaron dos lotes de aproximadamente $800 \mathrm{~m}^{2}$ con condiciones óptimas de drenaje donde se preparó el suelo con desbrozadora y rastra. Se aplicó el herbicida GOAL (Oxifluorfen) 15 días antes de la siembra con una dosis de 1.2 lt/ha.

Se utilizó un diseño de bloques completos al azar con siete repeticiones para las variables agronómicas y cuatro repeticiones para la variable nutricional Proteína cruda, bruta o total (PC). El tamaño de la unidad experimental fue de seis surcos de $5 \mathrm{~m}$ de largo por $0.7 \mathrm{~m}$ entre cada surco y una separación entre repeticiones de $1 \mathrm{~m}$. Los materiales fueron sembrados a semilla continua y raleados a 11 plantas por metro lineal.

Se realizó fertilización a la siembra con Fosfato diamónico (DAP) 36,1 kg/ha y Cloruro de potasio (KCL) con una dosis de $27,7 \mathrm{~kg} / \mathrm{ha}$. Se aplicaron $2 \mathrm{~kg} / \mathrm{ha}$ de elementos menores para complementar la fertilización, tomando en cuenta los resultados del análisis de suelos.

Tabla 2

Relación de genotipos de sorgo evaluados y sus características comerciales- Yopal 2016.

\begin{tabular}{|c|c|c|}
\hline $\begin{array}{c}\text { Numero de } \\
\text { Genotipo }\end{array}$ & $\begin{array}{c}\text { Nombre del } \\
\text { Genotipo }\end{array}$ & Características comerciales \\
\hline 1 & $600 \mathrm{D}$ & $\begin{array}{l}\text { Silero tipo Sudan, excelente capacidad de Rebrote. Puede Alcanzar } \\
75 \text { T/ha de producción de materia verde (Díaz et al., 2011; Tropical } \\
\text { Cis, 2014) }\end{array}$ \\
\hline 2 & ABS - 7000 & Sorgo forrajero x sudan (Anzu seed, 2012). \\
\hline 3 & Africano & $\begin{array}{l}\text { Sorgo tipo Sudan, excelente capacidad de rebrote y macollaje, resis- } \\
\text { tente a sequía, excelente sanidad de hoja, muy buena palatabilidad y } \\
\text { óptima conversión por alto contenido de azúcar (Tropical Cis, 2014). }\end{array}$ \\
\hline 4 & JJT-18 & $\begin{array}{l}\text { Sorgo forrajero colombiano, para suplementación animal, especial- } \\
\text { mente para épocas de sequía o de excesos de agua. Alto rendimientos } \\
\text { de forraje, con valores entre } 39 \mathrm{~T} / \text { ha a } 56 \mathrm{~T} / \text { ha de forraje verde para } \\
\text { corte a los } 90 \text { días después de emergencia (Bernal et al., 2014). }\end{array}$ \\
\hline
\end{tabular}


Pampa verde Sorgo Forrajero x Sudán fotosensible (No espiga durante los días largos del año). Útil para silo, pacas de heno, o corte verde (Anzu seed, 2016).

$6 \quad$ Pampa verde BMR-6

7
Pampa verde

Centurión
Sorgo tipo sudan $\mathrm{x}$ forrajero, maduración retardada, fotosensible, proteína superior al 14\%, sumamente digerible (Anzu seed, 2016).

Sorgo forrajero, recomendado para la siembra como silo, nervadura café, sorgo dulce, alto Contenido de Azúcares, hasta dos cortes, entre 50 a $60 \mathrm{TM} /$ corte/ha (Anzu seed, 2016).

Análisis estadísticos. Se realizó prueba de normalidad de Kolmogorov-Smirnov para las siete repeticiones y un análisis de varianza de un factor (ANOVA) para cada variable, con un valor de error de 0,05 . Posteriormente, se utilizó un Post-hoc con prueba de Tukey al 0,05 para los genotipos estudiados, con el fin de conocer cual de ellos es estadísticamente superior en cada variable. Las variables analizadas fueron las relacionadas con el rendimiento agronómico de forraje verde (T/ha) y materia seca (\%).

Modelo matemático. El modelo matemático para bloques completos al azar fue el siguiente:

$$
Y_{i j k}=U+T_{i}+B_{j}+E_{i j}
$$

$Y_{i j k}=$ Respuesta observada para el tratamiento i en la repetición $\mathrm{j}$

$U=$ Media general

$T_{i}=$ Efecto debido a tratamiento $\mathrm{i}$

$B_{j}=$ Efecto debido a bloque $\mathrm{j}$

$E_{i j}=$ Error experimental asociado al tratamiento i en la repetición $\mathrm{j}$

\section{Resultados}

\section{Etapa. Evaluación de Sostenibilidad del cultivo de Sorgo}

\section{a. Determinación de los puntos críticos}

Como primer hallazgo se muestra el resultado obtenido a partir de las entrevistas realizadas a los administradores de sistemas de producción bovina donde se elabora silo de Sorgo en explotaciones ubicadas en inmediaciones de la ciudad de Yopal, Casanare. Dichos resultados se plasman en la matriz DOFA (tabla 3) de acuerdo con la metodología descrita. 
Rios Moyano, D. K.; Conde Pulgarín, A.; Rios Moyano, C. F.

Tabla 3

Matriz DOFA entrevistas realizadas a administradores de sistemas de producción bovina donde se elabora silo de Sorgo ubicados en la ciudad de Yopal, Casanare

\section{FORTALEZAS}

- Buen rendimiento en toneladas por hectárea.

- Bajo costo de establecimiento y sostenimiento de cosecha de sorgo.

- Mejora el estado sanitario de los animales suplementados con el silo.

- Asegura el mantenimiento e incremento de ganancia de peso durante épocas de verano y/o sequía.

- Disminución de costos de producción destinados a la suplementación con alimento concentrado y suplementación de otro tipo, e inversión en manejos sanitarios curativos.

- Alto potencial de rebrote lo que ahorra el costo de semilla en tres a cuatro cosechas.

- El sorgo cuenta con alta tolerancia en suelos ácidos.

- A nivel ambiental acorta los días de los animales en etapa de ceba por lo que a su vez se disminuye la huella de gases efecto invernadero generados por cebas prolongadas.

- Alta precocidad del cultivo de sorgo.

- Apoya la recuperación de la biomasa del suelo.

- No requiere de adición de melaza para elaboración de silo

\section{OPORTUNIDADES}

- Obtención de ingresos adicionales en el modelo productivo por venta de silo a ganaderos.

- Incremento de la cultura de elaboración de bancos de alimentos para épocas de escasez, por ser un modelo innovador y la creciente necesidad de suplementar en épocas de verano.

- Incremento en capacidad de carga por medio de establecimiento de sistema semiestabulado.

- Disminución de muertes por enflaquecimiento progresivo y baja condición corporal.

- Menos animales enfermos por problemas nutricionales relacionados con desnutrición y desbalance energético.

- Vida útil de silo de sorgo prolongada hasta por un año.

- Políticas gubernamentales y gremiales de establecimiento de sistemas de alimentación alternativos que apoyan este tipo de iniciativas.

- Innovación en desarrollo de semillas para sorgo forrajero.

- Mantenimiento de producción de leche durante todo el año.

- Aprovechamiento de toda la planta, se disminuye el desperdicio 


\section{DEBILIDADES}

- Fumigación con piretroides de alta bioacumulación y alta residualidad.

- Poca innovación en tecnologías de manejo $y$ control de afectaciones fitosanitarias del sorgo.

- Se requiere abastecimiento de agua abundante para el segundo y tercer corte.

- Menor palatabilidad del silo por parte del ganado.

- Costos de fertilizantes en establecimiento y mantenimiento del cultivo de sorgo.

\section{AMENAZAS}

- Los rendimientos dependen del porcentaje de germinación y las condiciones agroclimáticas que en la actualidad tienen altísima variabilidad.

- Sistemas de conservación del silo deficientes que afectan la calidad por penetración de lluvias al silo.

- Falta de estandarización de la técnica de ensilado que puede afectar la conservación y calidad del silo.

- Requerimiento de maquinarias (tractor, remolque) e implementos agrícolas (sembradora abonadora, cortadora y cosechadora, entre otros) para el establecimiento del cultivo de sorgo.

- Contenido proteico de silo 9 a $12 \%$, dependiendo del genotipo y el tiempo de corte, éste es bajo en comparación a otros silos como el de maíz.

Con respecto a la determinación de los puntos críticos y la matriz DOFA (cuadro 3), en relación con las Fortalezas se pueden indicar que el cultivo de sorgo ofrece excelentes alternativas para la confección de ensilajes de alto rendimiento, alta calidad y seguridad en el cultivo (De León, 2007). El Sorgo produce en promedio 50 toneladas de ensilaje por hectárea al año, por su buen comportamiento agronómico y producción alta de biomasa (MinAgricultura, 2014).

Otras particularidades del Silo de Sorgo, son que no requiere la adición de melaza en su elaboración, tal como señala Garcés Molina et al. (2004), los ensilajes de maíz y de sorgo contienen suficiente cantidad de azúcares solubles y normalmente no requieren aditivos Además, con respecto al alto potencial de rebrote del sorgo, según Ledbetter, generará un mejor crecimiento para el pastoreo o cosechas múltiples (Ledbetter, 2019); también se señala que el sorgo brinda un alto potencial de producción de forraje de buena calidad, que puede ser conservado para su uso estratégico en cualquier momento y para distintos requerimientos nutricionales (De león, 2019). El sorgo, es un cultivo agronómicamente perfecto ya que genera una alta producción con un consumo mucho menor de agua que los otros cereales de verano (Carrasco, Zamora y Melín, 2011). Dentro de las debilidades del establecimiento del cultivo se resaltan el hecho de las aplicaciones constantes de agroquímicos piretroides para el control de insectos altamente limitantes como Spodoptera frugiperda y Diatraea saccharalis (Flores, 2010) y la menor palatabilidad del silo de sorgo en los animales. Las Amenazas relevantes para la zona obedecen a la falta de capacitación que actualmente existe para la estandarización adecuada de procesos de ensilaje, así como el correcto uso de planes de manejo agronómico que aseguren que la productividad del cultivo se dará de la forma adecuada, así mismo, se considera que los contenidos proteícos del sorgo son bajos en comparación con 
Rios Moyano, D. K.; Conde Pulgarín, A.; Rios Moyano, C. F.

los ofrecidos por otro tipo de forrajes directos o cultivos que pueden ser utilizados para la elaboración de silos.

\section{b. Selección de indicadores.}

A continuación, se presentan los indicadores de sostenibilidad de Bancos de alimentación ganadera de silo de sorgo como alternativa para nutrición bovina, en bosque húmedo tropical bh-T en Yopal Casanare (tabla 4).

Tabla 4

Indicadores generados para determinar el enfoque de sostenibilidad

\begin{tabular}{|c|c|c|c|c|c|}
\hline \multirow{2}{*}{\multicolumn{2}{|c|}{$\begin{array}{c}\text { Dimensión y aporte / } \\
\text { Tema }\end{array}$}} & \multirow{2}{*}{ Subtema } & \multirow{2}{*}{ Indicador } & \multicolumn{2}{|c|}{ Likert (1 - 5) } \\
\hline & & & & Obtenido & Ideal \\
\hline \multirow{13}{*}{ 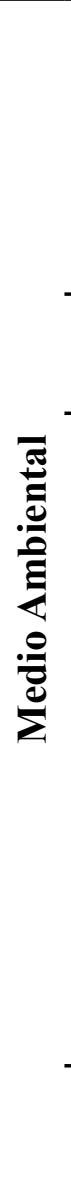 } & & Cambio climático & $\begin{array}{l}\text { Emisión de gases efecto } \\
\text { invernadero }\end{array}$ & 5 & 5 \\
\hline & Atmósfera & Captura de carbono & Biomasa & 4 & 5 \\
\hline & & Calidad del aire & $\begin{array}{l}\text { Concentración de contami- } \\
\text { nación atmosférico }\end{array}$ & 3 & 5 \\
\hline & Suelo & Agricultura & $\begin{array}{l}\text { Área permanente de } \\
\text { cultivos }\end{array}$ & 5 & 5 \\
\hline & \multirow{7}{*}{ Biodiversidad } & Ecosistema & Estructura ecosistémica & 4 & 5 \\
\hline & & & Conservación de los recur- & & \\
\hline & & Ecosistema & sos naturales, agua, suelo, & 3 & 5 \\
\hline & & $\begin{array}{l}\text { Cuidado de la biodiver- } \\
\text { sidad }\end{array}$ & $\begin{array}{l}\text { Área protegida en \% de } \\
\text { área total }\end{array}$ & 4 & 5 \\
\hline & & Especies & $\begin{array}{l}\text { Abundancia de especies } \\
\text { introducidas }\end{array}$ & 2 & 5 \\
\hline & & Cantidad de agua & $\begin{array}{l}\text { Extracción de agua subte- } \\
\text { rránea anual }\end{array}$ & 4 & 5 \\
\hline & & Regulación del agua & Protección & 4 & 5 \\
\hline & \multirow{2}{*}{\multicolumn{3}{|c|}{$\begin{array}{r}\text { Total } \\
\text { Enfoque de sostenibilidad Ambiental }\end{array}$}} & 38 & 50 \\
\hline & & & & $76 \%$ & $100 \%$ \\
\hline
\end{tabular}




\begin{tabular}{|c|c|c|c|c|c|}
\hline \multirow{6}{*}{$\begin{array}{l}\bar{\pi} \\
\frac{\pi}{0} \\
\mathscr{0}\end{array}$} & Salud & Sanitarios & $\begin{array}{l}\text { Manejo de empaques de } \\
\text { agroquímicos }\end{array}$ & 4 & 5 \\
\hline & Bienestar & Calidad de vida & $\begin{array}{l}\% \text { Ingresos que apoyen el } \\
\text { sistema productivo }\end{array}$ & 4 & 5 \\
\hline & $\begin{array}{l}\text { Seguridad y Sobera- } \\
\text { nía alimentaria }\end{array}$ & Estado nutricional & $\begin{array}{l}\text { \% Satisfacción de necesi- } \\
\text { dades en dieta alimenticia } \\
\text { adecuada }\end{array}$ & 3 & 5 \\
\hline & \multirow[t]{3}{*}{ Organización } & Asociatividad & $\begin{array}{l}\text { \% participación gremial y/o } \\
\text { asociatividad en estableci- } \\
\text { miento e implementación } \\
\text { de silo de sorgo }\end{array}$ & 3 & 5 \\
\hline & & & Total & 14 & 20 \\
\hline & & \multicolumn{2}{|c|}{ Enfoque de sostenibilidad Social } & $70 \%$ & $100 \%$ \\
\hline \multirow{10}{*}{ 异 } & \multirow{3}{*}{$\begin{array}{l}\text { Estructura econó- } \\
\text { mica }\end{array}$} & Generación de empleo & Tasa de desempleo & 4 & 5 \\
\hline & & Comercio & $\begin{array}{l}\text { Balance de comercio en } \\
\text { bienes y servicios }\end{array}$ & 4 & 5 \\
\hline & & Nivel financiero & $\begin{array}{l}\text { Líneas especiales de Crédi- } \\
\text { to LEC recursos }\end{array}$ & 3 & 5 \\
\hline & \multirow{7}{*}{$\begin{array}{c}\text { Patrones de } \\
\text { consumo y produc- } \\
\text { ción }\end{array}$} & Consumo de material & $\begin{array}{l}\text { Intensidad uso de materia- } \\
\text { les }\end{array}$ & 4 & 5 \\
\hline & & \multirow{2}{*}{ Uso de energía } & $\begin{array}{l}\text { Consumo de energía reno- } \\
\text { vable año }\end{array}$ & 3 & 5 \\
\hline & & & Consumo de energía anual & 2 & 5 \\
\hline & & \multirow{2}{*}{$\begin{array}{l}\text { Manejo y generación de } \\
\text { residuos }\end{array}$} & $\begin{array}{l}\text { Generación industrial de } \\
\text { residuos }\end{array}$ & 5 & 5 \\
\hline & & & $\begin{array}{l}\text { Generación de residuos pe- } \\
\text { ligrosos }\end{array}$ & 3 & 5 \\
\hline & & & Total & 28 & 40 \\
\hline & & \multicolumn{2}{|c|}{ Enfoque de sostenibilidad Económico } & $70 \%$ & $100 \%$ \\
\hline
\end{tabular}




\begin{tabular}{|c|c|c|c|c|c|}
\hline \multirow{6}{*}{ 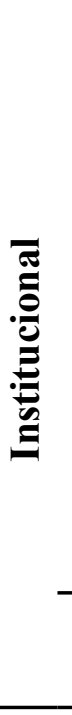 } & \multirow{6}{*}{ Marco institucional } & $\begin{array}{l}\text { Implementación de es- } \\
\text { trategias de desarrollo } \\
\text { sostenible }\end{array}$ & $\begin{array}{l}\text { Estrategias de desarrollo } \\
\text { sostenible }\end{array}$ & 4 & 5 \\
\hline & & $\begin{array}{l}\text { Cooperación institucio- } \\
\text { nal }\end{array}$ & $\begin{array}{l}\text { Implementación de acuer- } \\
\text { dos o convenios globales } \\
\text { ratificadas }\end{array}$ & 4 & 5 \\
\hline & & Ciencia y tecnología & $\begin{array}{l}\text { Gastos en investigación y } \\
\text { desarrollo en \% PIB }\end{array}$ & 2 & 5 \\
\hline & & $\begin{array}{l}\text { Preparación y respuesta } \\
\text { a los desastres naturales }\end{array}$ & $\begin{array}{l}\text { Pérdidas humanas económi- } \\
\text { cas por desastres naturales }\end{array}$ & 4 & 5 \\
\hline & & & Total & 14 & 20 \\
\hline & & Enfoque de & sostenibilidad institucional & $70 \%$ & $100 \%$ \\
\hline \multirow{13}{*}{ 苞 } & & Nutrición & \% Aplicación fertilizantes & 3 & 5 \\
\hline & Agrícola & Fitosanidad & $\begin{array}{l}\text { \% Aplicación insecticidas, } \\
\text { fungicidas y herbicidas }\end{array}$ & 3 & 5 \\
\hline & & Propagación & $\begin{array}{l}\text { \% Disponibilidad semillas } \\
\text { en el mercado }\end{array}$ & 4 & 5 \\
\hline & \multirow{10}{*}{ Pecuario } & Nutrición & $\begin{array}{l}\text { \% Fibra Digestible Neutra y } \\
\text { Fibra digestible ácida }\end{array}$ & 3 & 5 \\
\hline & & $\begin{array}{l}\text { Manejo sistema explo- } \\
\text { tación }\end{array}$ & $\begin{array}{l}\text { \% Implementación sistema } \\
\text { semiestabulado }\end{array}$ & 3 & 5 \\
\hline & & \multirow{2}{*}{ Reproducción } & Disminución días abiertos & 4 & 5 \\
\hline & & & $\%$ Tasas de preñez & 2 & 5 \\
\hline & & \multirow{4}{*}{ Sanidad } & $\begin{array}{l}\text { Generación industrial de } \\
\text { residuos }\end{array}$ & 5 & 5 \\
\hline & & & $\%$ Mortalidad & 4 & 5 \\
\hline & & & $\begin{array}{l}\text { Utilización de medicamen- } \\
\text { tos para tratamientos veteri- } \\
\text { narios }\end{array}$ & 4 & 5 \\
\hline & & & $\%$ enfermedades & 3 & 5 \\
\hline & & Bienestar animal & $\begin{array}{l}\text { Cinco libertades (hambre } \\
\text { y sed, de incomodidad, de } \\
\text { lesión, dolor o enfermedad, } \\
\text { de expresar un comporta- } \\
\text { miento natural y de miedo y } \\
\text { angustia) }\end{array}$ & 4 & 5 \\
\hline & & \multicolumn{2}{|c|}{ Enfoque de sostenibilidad Económico } & $\begin{array}{c}28 \\
70 \%\end{array}$ & $\begin{array}{c}40 \\
\mathbf{1 0 0} \%\end{array}$ \\
\hline
\end{tabular}


Es así como, en términos generales se determinó por medio de la evaluación realizada en los indicadores de sostenibilidad propuestos para este trabajo, que el silo de sorgo es medianamente sostenible ya que se obtiene un promedio en las cinco dimensiones evaluadas de $73 \%$ de cumplimiento del 100\%, de los cuales el de mayor porcentaje de cumplimiento corresponde a las dimensiones medio ambiental y técnica $(76 \%)$.

La dimensión Medio Ambiental, obtiene un porcentaje de cumplimiento de $76 \%$, donde los indicadores que afectan esta dimensión son: concentración de contaminación atmosférico, conservación de los recursos naturales y abundancia de especies introducidas. Sin embargo, indicadores relacionados con emisión de gases efecto invernadero, acumulación de biomasa y manejo de recurso hídrico fortalecen dicha dimensión. El sorgo es una buena opción, porque como cultivo permite utilizar menos agua tolerando periodos de sequía, de acuerdo con Abebe et al. (2020) el sorgo es uno de los cereales mas dominantes tanto social como agrícolamente especialmente en zonas secas además, Romero (2019) considera es un cultivo ideal para integrar un sistema sustentable de producción, como alternativa de rotación, por la cantidad de rastrojo residual, por su extendido sistema radicular y por su sanidad diferencial.

En cuanto a la dimensión social, se ponderó un porcentaje de $70 \%$ de cumplimiento en su enfoque de sostenibilidad, donde los indicadores estado nutricional y asociatividad afectan la respuesta de la dimensión. Morell, Exposito y Ruiz (2018) indican que al no existir una tradición en el cultivo su desarrollo se limita, si se logra aumentar las áreas sembradas y producción, se incrementan los empleos durante las labores del cultivo, contribuyoendo a mayores ganancias y mejoramiento de las condiciones y calidad de vida de los productores y sus familias.

A nivel económico se obtuvo un $70 \%$ de sostenibilidad lo que se debe a los valores de los indicadores de nivel financiero, uso de energía y manejo y generación de residuos, aunque como señala Rocamundi (2007) el sorgo se presenta como una excelente alternativa para desarrollar sistemas productivos eficientes y rentables, la falta de lineas de credito especializado puede influir en la implementación de los sistemas de producción agrícola.

En la dimensión Institucional, se obtuvo un 70\% de sostenibilidad donde el indicador ciencia y tecnología influye de formar directa en el resultado. El gasto en investigación y desarrollo, es muy bajo en región de América Latina donde del Producto Interno Bruto - PIB es del 0,8\% en en promedio se invierte en este aspecto, pero en Colombia el valor está por debajo de 0,5\% (La República, 2019). Esto se puede asociar con que se deben sostener programas públicos y privados de mejoramiento genético específico que permitan disponer de materiales genéticos con mejoras en su adaptación ambiental (Romero, 2019), y planes de extensión que faciliten su adopción tecnológica; así como, mayores apoyos financieros, representados en créditos con tasas de interés competitivas que promuevan el desarrollo tecnológico y la integración de la agricultura con la ganadería

Con respecto a la dimensión técnica se obtuvo un porcentaje de $76 \%$, siendo este medianamente sostenible, mostrando solamente en el indicador porcentaje de tasas de preñez un valor de 2 (Escala de Likert) y valores superiores a 3 en los demás indicadores; esto puede tener su origen en la falta de experiencia para manejo tecnológico del cultivo (Morell, Exposito y Ruiz, 2018), requiriendo así una mayor conciencia en cuanto a la importancia y oportunidad para los productores de contar con una oferta 
tecnológica que bajo unas buenas condiciones de manejo y producción les va a permitir tener un forraje de alta calidad nutricional y buena productividad (MinAgricultura, 2014). Además, se requiere mayor capacitación en cuanto a los puntos críticos durante las etapas de establecimiento, crecimiento, manejo postcosecha, almacenamiento y conservación del forraje (Morell, Exposito y Ruiz, 2018); así como, de su uso estratégico como suplemento a la dieta de acuerdo con los estados fisiológicos en los cuales de utiliza este recurso forrajero en la alimentación en los diversos sistemas de producción bovina.

\section{Etapa. Evaluación del rendimiento de genotipos de sorgo}

Rendimiento en fresco (T/ha). Al evaluar esta variable y corroborar su distibución normal (prueba de Kolmogorov-Smirnov sig $=0,2$ ), se encontró que existen diferencias estadísticas al realizar el ANOVA ( $p$ valor $=0,05)$ en la variable rendimiento en fresco $(\mathrm{T} / \mathrm{ha})$ por parte de los genotipos en evaluación (ver tablas 5 y 7), donde el genotipo con el mayor promedio de peso fue Pampa Verde (69,26 T/ha) presentando diferencias estadísticas con los genotipos de menor rendimiento de peso en fresco Pampa Verde BMR-6, Pampa Verde Centurión, Africano y JJT-18, con 50,02 , 52,36 , 56,75 y 57,14 T/ha, respectivamente (ver tabla 5). Cabe resaltar que se estimó el peso de la parcela cosechada en toneladas para tener un aproximado en un área representativa de cultivo.

Tabla 5

ANOVA para la variable peso fresco T/ha en siete genotipos de sorgo. Yopal, $2016 B$.

\begin{tabular}{lccccc}
\hline $\begin{array}{l}\text { Fuente de } \\
\text { variación }\end{array}$ & $\begin{array}{l}\text { Grados de } \\
\text { libertad }\end{array}$ & $\begin{array}{l}\text { Suma de } \\
\text { cuadrados }\end{array}$ & $\begin{array}{l}\text { Cuadrado } \\
\text { Medio }\end{array}$ & $\begin{array}{l}\text { F calcula- } \\
\text { da }\end{array}$ & $\begin{array}{l}\text { P(valor } \\
\text { alfa=0.05) }\end{array}$ \\
\hline Genotipo & 6 & 1544,02 & 257,34 & 6,40 & $0,00^{*}$ \\
Bloque & 6 & 3684,17 & 614,03 & 15,27 & 0,00 \\
Error & 28 & 1125,80 & 40,21 & & \\
\hline Total & 41 & 147489,54 & & & \\
*Diferencias significativas al nivel del 0,05 &
\end{tabular}

Porcentaje de materia seca (MS \%). Al corroborar su distibución normal (prueba de Kolmogorov-Smirnov sig $=0,15)$ y al obtener el ANOVA ( $p$ valor $=0,05)$, se encontró que éste componente de evaluación no mostró diferencias estadísticas entre los siete genotipos en evaluación (ver tablas 6 y 7). Se destaca que el genotipo JJT-18 tuvo el mayor porcentaje de acumulación de materia eca con 23,07 \% seguido por Africano con $21,84 \%$, genotipos que a su vez presentan promedios de peso fresco de 57,14 T/ha y 56,75 T/ha, respectivamente. El genotipo Pampa Verde presentó un desempeño más bajo en acumulación de materia seca con 15,88 \%, sin embargo, es el genotipo de mayor volumen de producción en peso fresco (69,26 T/ha), por lo cual se asume que es un buen acumulador de líquidos mas no de tejido seco. 
Tabla 6

ANOVA para la variable porcentaje de materia seca en siete genotipos de sorgo. Yopal, $2016 B$.

\begin{tabular}{cccccc}
\hline $\begin{array}{c}\text { Fuente de } \\
\text { variación }\end{array}$ & $\begin{array}{l}\text { Grados de } \\
\text { libertad }\end{array}$ & $\begin{array}{l}\text { Suma de } \\
\text { cuadrados }\end{array}$ & $\begin{array}{l}\text { Cuadrado } \\
\text { Medio }\end{array}$ & $\begin{array}{l}\text { F calcula- } \\
\text { da }\end{array}$ & $\begin{array}{l}\text { P(valor } \\
\text { alfa=0.05) }\end{array}$ \\
\hline Genotipo & 6 & 77,09 & 12,85 & 0,60 & $0,72^{\mathrm{NS}}$ \\
Bloque & 4 & 360,84 & 90,21 & 4,22 & 0,02 \\
Error & 16 & 342,26 & 21,39 & & \\
\hline Total & 27 & 11310,96 & & & \\
\hline
\end{tabular}

${ }^{\text {NS }}$ Diferencias no significativas al nivel del 0,05

Tabla 7

Promedio y prueba de comparación de medias HSD Tukey* para las variables tonelada por hectárea (T/ha) y porcentaje de materia seca (MS\%) en siete genotipos de sorgo Yopal, $2016 B$.

\begin{tabular}{ccc}
\hline Genotipo & T/ha & MS \% \\
\hline 600 D & $59,44 \mathrm{ABC}$ & $18,66 \mathrm{~A}$ \\
ABS - 7000 & $66,95 \mathrm{BC}$ & $18,72 \mathrm{~A}$ \\
Africano & $56,75 \mathrm{AB}$ & $21,85 \mathrm{~A}$ \\
JJT-18 & $57,14 \mathrm{AB}$ & $23,07 \mathrm{~A}$ \\
Pampa verde & $69,26 \mathrm{C}$ & $15,88 \mathrm{~A}$ \\
Pampa verde BMR-6 & $50,02 \mathrm{~A}$ & $19,57 \mathrm{~A}$ \\
Pampa verde Centurión & $52,36 \mathrm{~A}$ & $18,02 \mathrm{~A}$ \\
Desviación estándar & 10,13 & 5,69 \\
\hline
\end{tabular}

*Letras diferentes indican diferencias estadísticas entre tratamientos al nivel del 0,05 
Rios Moyano, D. K.; Conde Pulgarín, A.; Rios Moyano, C. F.

\section{Discusión de resultados}

\section{Etapa. Evaluación de Sostenibilidad del cultivo de Sorgo}

\section{a. Determinación de los puntos críticos.}

Se puede afirmar que el Sorgo asegura el mantenimiento e incremento de ganancia de peso durante épocas de verano y/o sequía, ya que los animales alimentados con ensilajes de sorgo logran niveles respuesta productiva similares a los obtenidos con ensilajes de maíz; ya que, en ganancia de peso, la respuesta animal presenta resultados compatibles con procesos de recría y de engorde de alta eficiencia y equipara los índices de dietas basadas en maíz (De león, 2019). El sorgo cuenta además con una elevada capacidad de rebrote en algunos genotipos, con posibilidad de segundo corte o pastoreo de la fracción vegetativa y bajo costo de implantación, es así como este se constituye en una alternativa válida y crecientemente empleada (Torecillas, 2006).

El cultivo de sorgo se desarrolla muy bien cuando las temperaturas son altas, adaptándose a regiones de clima cálido en Colombia con temperaturas entre $21^{\circ} \mathrm{C}$ y $30^{\circ} \mathrm{C}$ y alturas comprendidas entre el nivel del mar y los 1200 metros de altitud. El sorgo se puede desarrollar bien en una amplia gama de suelos, desde los arenosos hasta los arcillosos. Los mayores rendimientos se obtienen en suelos de textura franca y sus afines: franco arcilloso, franco limosos y franco arenosos, bien drenados y libres de inundaciones ya que condiciones demasiado húmedas limitan notablemente su producción. El sorgo posee un amplio rango de adaptación tanto a la acidez como a la alcalinidad de los suelos. Crece bien en lotes cuyo $\mathrm{pH}$ oscila entre 5,5 y 6,5. Igualmente, el sorgo tiene la particularidad de aportar elevadas cantidades de rastrojo que contribuyen a mejorar la cobertura de los suelos (CIAT, 1990; Fenalce, 2010; Carrasco, Zamora y Melín, 2011).

Así mismo, se pueden asociar algunas oportunidades del silo de sorgo al desarrollo de éste cultivo alternativo para la región con el hecho de su potencial de rendimiento y calidad, que permiten aumentar la carga animal, sin disminuir las respuestas individuales y además mejorar la utilización de las pasturas durante su ciclo de crecimiento, asegurando contar con el forraje necesario durante todo el año (De León, 2014; Romero, 2019). Entonces, el sorgo (Sorghum bicolor L. Moench.) es considerado como otra alternativa de alimento conservado mediante ensilaje para el ganado bovino, ya que es altamente productivo, nutritivo y se puede producir a menores costos (Contexto Ganadero, 2018). Además, la implementación y la incorporación de los ensilajes de sorgo, contribuye a la intensificación de los sistemas ganaderos debido a su alto potencial de producción de forraje de buena calidad. Estos atributos de los ensilajes, tienen un efecto directo en el resultado productivo y económico de las empresas ganaderas. Además, con la incorporación del sorgo, se incrementaará significativamente la cantidad y calidad de forraje disponible en el sistema; esto, no solo permite cubrir la demanda de forraje y mantener la condición corporal de los vientres sino también brinda la posibilidad de implementar otras estrategias productivas como el ciclo completo. Los mayores rendimientos obtenidos redundan finalmente en un menor costo del $\mathrm{kg}$ de materia seca producida (Giménez et al., 2018). 
Es importante recalcar, que existe una importante oferta de híbridos comerciales de sorgo en el mercado (Diez y Sardiña, 2012) gracias a la innovación en desarrollo de semillas para sorgo forrajero, este nos ofrece variabilidad en lo que a "tipos" se refiere (sudan, graniferos e híbridos), lo que significa una buena oferta según los diversos objetivos y manejos de los productores (Fassio et al., 2002), la amplitud de materiales genéticos en sorgo ofrece excelentes alternativas para la confección de ensilajes de alto rendimiento, calidad y seguridad (De león, 2019).

Otra aptitud potencial del sorgo para elaboración de silo, se refiere al aprovechamiento de toda la planta, disminuyendo el desperdicio ya que la forma más corriente de aprovecharla es mediante su ensilaje, además, este recurso es una alternativa de alimentación para rumiantes que puede ser consumida por cualquier categoría animal y en cualquier estado fisiológico (Carrasco, Zamora y Melín, 2011; Barbosa, 2012).

Ahora bien, dentro de los aspectos desfavorables del establecimiento del ensilaje de sorgo se destaca que desde el punto de vista nutricional presenta limitantes en lo que respecta al contenido de proteína (Carrasco, Zamora y Melín, 2011). Otra de las desventajas del silo de Sorgo, es que el grano en estado duro es aprovechado con baja eficiencia por parte del sistema digestivo de los animales, es decir que una parte del mismo pasa sin ser digerido (Kent, 2019).

Se requieren conocimientos para el manejo agronómico del cultivo de sorgo, riesgos climáticos durante el ciclo del cultivo, riesgo de fallas en la confección del ensilaje, cuidado y mantenimiento de la integridad de la bolsa durante el almacenamiento, extracción y suministro del silo (Ayala, 2010).

En cuanto al manejo agronómico es importante resaltar que el uso excesivo de insecticidas como piretroides pueden convertirse en un problema de salud publica, debido alto uso de los mismos generando un impacto en la salud humana y el medio ambiente, siendo altamente tóxicos en peces e invertebrados (Devine et al., 2008; Varona et al., 2012)

\section{b. Selección de indicadores.}

El uso de los cultivos forrajeros en los sistemas agropecuarios desde hace aproximadamente 1.300 años a.C., ha contribuido para que el ganado sea comparativamente menos vulnerable a los ambientes adversos y cambios climáticos (Iqbal et al., 2015). De acuerdo con los indicadores de sostenibilidad analizados para el cultivo de sorgo forrajero los de mayor ponderación son el Medio Ambiental y el Técnico, con un 76\% de cumplimiento para cada uno de ellos, por tanto es importante abordar características especiales del cultivo tal como mencionan Janssen et al. (2015), quienes afirman que el sorgo dulce es considerado uno de los cultivos más eficientes para convertir el $\mathrm{CO}_{2}$ atmosférico en azúcar, lo que lo convierte en un cultivo prometedor para la bioenergía al tiempo que satisface las necesidades de alimentos y forrajes.

Iqbal et al. (2015) destacan que la semilla de sorgo forrajero es más económica que la de maíz forrajero, así mismo el maíz forrajero requiere más fertilizantes, especialmente urea, mientras que el sorgo forrajero tiene el potencial de producir un rendimiento de forraje verde razonablemente alto con un menor uso de fertilizantes. El sorgo forrajero requiere menos riego que el maíz forrajero, ya que el sorgo forrajero es más eficiencia en el uso del agua por su menor transpiración debido a la presencia de una 
cutícula gruesa en las hojas y en el tallo; soportando así períodos de escacez hídrica manteniendo una alta productividad con sistemas de riego limitados en comparación con el maíz además, puede reemprender su crecimiento posterior a un periodo de sequía prolongado (Pérez et al., 2010; Iqbal et al., 2015; Pino y Heinrichs, 2017). Lo anterior concuerda con lo reportado por Saeed y El-Nadi (1998) quienes concluyen que las plantas de sorgo regadas en abundancia y con poca frecuencia habían reducido la altura de los tallos, la acumulación de biomasa y la eficiencia en el uso del agua; por el contrario, el riego ligero y frecuente aumentó estos parámetros.

El sistema ganadero utilizado actualmente en la Orinoquía Colombiana, se caracteriza por contar con tecnologías que incluyen ganadería semiextensiva, pastoreo rotacional, quema dos veces al año, acceso directo a fuentes de agua ó bebederos, lo anterior se relaciona de forma directa con el conflicto ambiental que incluye competencia por recurso hídrico, erosión, pérdida de biodiversidad y praderas degradadas (Bustamante y Rojas-Salazar, 2018); una herramienta para contrarrestras los efectos de este tipo de sistemas ganaderos es la elaboración de silos a partir de cultivos de sorgo, ya que permite evitar la degradación de praderas y la pérdida de la biodiversidad por la apertura de mayores áreas para el pastoreo directo de los bovinos.

Es importante tener en cuenta que el uso de energía para el caso del sorgo tiene asignación de valores bajos de sustentabilidad (menores a 3), los cuales como mencionan Zinck et al. (2005) deben ser corroborados en balances energéticos realizados con entrevistas múltiples y mediciones directas in-situ, tales como determinación del rendimiento del cultivo utilizando la técnica del corte directo en la parcela del agricultor.

\section{Etapa. Evaluación del rendimiento de genotipos de sorgo}

Los resultados tonelada por hectárea (T/ha) para los siete genotipos evaluados, mostraron un promedio de 58,64 T/ha; un rendimiento superior con respecto al reportado por Cabrales, Montoya y Rivera (2007) con el genotipo Blanco Criollo 40,03 T/ha de forraje verde que fue el que obtuvo la mayor producción en evaluaciones realizadas en la costa Atlántica de Colombia; por su parte Ruz-Reyes, Escalona-Peña y Romero-Arias (2018) reportaron rendimientos de 68,8 T/ha en el genotipo CIAP 2E-95 y 66,8 T/ha para el genotipo CIAP 29, superando los rendimientos medios obtenidos pero similares a los genotipos ABS - 7000 y Pampa verde. Evaluaciones realizadas por Díaz et al., (2011) en cultivares de sorgo para silo en Argentina indican una producción promedio de materia verde de 54,3 T/ha, con un máximo de 86,2 T/ha (Genotipo Surargraze) y un mínimo de 37,1 T/ha (Genotipo Gran Silo).

Según Valente (1992 citado por Ribeiro, Rodríguez y Gonçalves, 2007) la producción mínima de sorgo para que sea viable económicamente es de 40 toneladas de masa verde por hectárea; aunque estos resultados son inferiores a las logradas con prácticas de fertilización adecuadas y usando semillas certificadas de alta calidad en las que se han logrado hasta 60 ton/FV/ha en primer corte, el valor de la fracción proteica fluctúa entre $9 \%$ y $12 \%$ en estado de grano pastoso, momento en el que se produce la cosecha para ensilaje. Está última variable nutricional es la condición por la que no puede utilizarse en la alimentación de los rumiantes como fuente proteica (Contexto Ganadero, 2018). Es importante señalar que, en evaluaciones realizadas, a la edad de 80 días se obtuvo una producción de biomasa verde del 
sorgo forrajero superior en $42 \%$ a la del maíz y en $25 \%$ al sorgo comercial en cuanto a la producción de forraje en verde y seco (MinAgricultura, 2014).

Con respecto a la materia seca (MS) promedio obtenida de la evaluación de los siete genotipos fue de 19,69 \%, la cual es similar a la obtenida en estudios desarrollados por Vargas (2005), donde el porcentaje de MS para 15 genotipos fue en promedió 14,06 \%; con un valor máximo de 15,44\% para los genotipos CIAT 610 y un valor mínimo de 11,94 \% para CIAT 496. En el caso de Serra et al. (2008) reportan porcentaje de materia seca con valores entre 28,5\% para la variedad Supergrazer y mínimos de 15,9 \% para la variedad Teide. El contenido en MS en evaluaciones realizadas por Resch et al. (2013) varió de $17,9 \%$ en el primer corte hasta $29,5 \%$ en el último corte. Por su parte Corral-Luna et al. (2011) encontraron que dentro de los sorgos de nervadura café, el contenido de MS tuvo un promedio de $24,7 \%$, siendo menores que los reportados para ensilajes de maíz, los cuales están entre 26,9\% y 36,8\%.

Para Chao-Chen, Xiao-Lin y Guang-Hui (2018) el sorgo dulce, es una alternativa potencial al maíz en la llanura del norte de China. como cultivo bioenergético y forrajero tolerante a la sequía y a salinidad de los suelos. Como cultivo forrajero es una alternativa importante al maíz, por su tolerancia a sequía, altas temperaturas ó retrasos en la siembra (Oliver et al., 2004). Esto reafirma la necesidad de un uso sostenible de cultivos agrícolas locales tolerantes a sequía y que sirvan de fuente para la alimentación humana y la alimentación animal (Milesi, 2016).

La diversificación de la producción y la integración de cultivos y ganado en todos los niveles, desde la explotación hasta el paisaje, la comunidad, el territorio y la región, contribuirán a fortalecer la resiliencia y mejorar la eficiencia en la utilización de recursos, donde los recursos genéticos son un activo fundamental para el desarrollo agrícola sostenible (FAO, 2016), por tanto se considera que los ensayos de genotipos de sorgo promisorios para la región ganadera de Colombia son escenciales ya que permiten que los sistemas de producción cuenten con alternativas sostenibles de cultivos para la alimentación de rumiantes, los cuales permitan suplir las necesidades alimenticias en épocas de sequía.

\section{Conclusiones}

A través de los métodos teóricos y empíricos utilizados para la evaluación técnica y de sostenibilidad del sorgo se resalta que el cultivo es un gran apoyo para los productores ya que se convierte en un cultivo alternativo para la alimentación de rumiante, que genera un beneficio en los sistemas ganaderos de la llanura Colombiana. Desde la dimensión ambiental de la sostenibilidad el establecimiento de cultivo de sorgo y elaboración de silo es favorable en los sistemas de producción ganaderos debido a que disminuye la huella de carbono. En cuanto a la dimensión de sostenibilidad social el modelo productivo de silo de sorgo para alimentación bovina tiene un nivel medio de favorabilidad requiriendo mayor vinculación y participación de mujeres y jóvenes de manera efectiva y que exista un apoyo para aumentar la cultura de siembra del cultivo. La dimensión económica se categorizó como medianamente sostenible, atribuyéndolo a los costos fijos del establecimiento y sostenimiento del cultivo son relativamente altos debido al paquete tecnológico con uso de maquinaria y mano de obra. La dimensión institucional y gremial requiere fortalecimiento en aspectos de extensión, recursos de capital con bajos intereses y programas de promoción para la integración eficiente y sostenible de la agricultura con la ganadería. Finalmente, la dimensión técnica de la integración del cultivo de sorgo requiere mayor investigación de semillas y su 
Rios Moyano, D. K.; Conde Pulgarín, A.; Rios Moyano, C. F.

adaptación en los diferentes agroecosistemas de la Orinoquia; no obstante, el valor encontrado en esta dimensión da cuenta de una alternativa favorable para la sostenibilidad general en la ganadería bovina de la región. Adicionalmente, la evaluación del rendimiento de los siete genotipos comerciales de sorgo mostró que en forraje verde se obtienen valores entre las 50,02 y 69,26 T/ha y porcentajes de materia seca de entre 15,88 y $23,07 \%$, mostrando una respuesta adecuada a nivel de rendimiento para la zona. Por tanto, se infiere que el sorgo es un cultivo promisorio, para la intensificación sustentable de la producción de carne en la región de la Orinoquia en especial bajo las condiciones de bosque húmedo tropical, el cual puede ser utilizado como suplemento en la alimentación de explotaciones bovinas.

\section{Agradecimientos}

Para desarrollar el presente trabajo se contó con la financiación de la Universidad de La Salle, Vicerrectoría de Investigación y Transferencia - VRIT, en el marco de la convocatoria interna 2016.

A los estudiantes del Proyecto Educativo Utopía de La Universidad de La Salle Colombia, Línea Productiva de Cereales y Leguminosas, por su contribución en el trabajo de campo. Adicionalmente se extiende el agradecimiento al Dr. Rodrigo Gonzales director del Centro de Investigación y Capacitación - CIC- de la Universidad de La Salle con sede en Yopal, Colombia, por responder las preguntas de la entrevista realizada sobre: sistemas de explotación ganadera en Y opal.

Los autores aclaran que en el presente artículo no existe conflicto de interés con la publicación de este manuscrito.

Para acceder a la información sobre los datos utilizados en el presente artículo, estos se encuentran disponibles previa solicitud por email al autor principal del documento.

\section{Referencias}

Abebe, A., Aemiro, B., Getawey, G., Tesfaye, A. \& Alemu, L. (2020). Evaluation of sorghum (Sorghum bicolor (L.) Moench) variety performance in the lowlands area of wag lasta, north eastern Ethiopia. Cogent Food \& Agriculture, 6(1), 2-12. https://doi.org/10.1080/23311932.2020.1778603

Anzu Seed. (2012). ABS 7000. Semilla Certificada. Recuperado el 26 de febrero de 2021 de http:// www.pampaverde.com/abs 7000.html

Anzu Seed. (2016). Sorgo forrajero. Una línea de sorgos con propósito. Recuperado el 26 de febrero de $2021 \mathrm{de}$ http://www.pampaverde.com/pampaverde bmr6.html

Ayala, W. (2010). Ensilaje de grano húmedo de sorgo. Institución Nacional de Investigación Agropecuaria - INIA. Uruguay. Mayo de 2010. Recuperado el 24 de febrero de 2021 de http://www.ainfo.inia.uy/ digital/bitstream/item/574/1/14445240610133839.pdf

Barbosa, J. (2012) Mejoramiento de la producción de leche mediante nuevas alternativas alimenticias en la Hacienda El Caribe adscrita a la empresa ganadería el 24, en Fundación - Magdalena. Recuperado el 24 de febrero de 2021 de http://repositorio.ufpso.edu.co:8080/dspaceufpso/bitstream/123456789/237/1/25216.pdf 
Productividad y sostenibilidad del cultivo de sorgo forrajero como alternativa para la alimentación de rumiantes

Bernal, J. H., Rincón, A., Guevara, E. J., Hernández, R. S. \& Flórez, H. (2014). Sorgo forrajero JJT-18. Boletín Técnico. Corporación Colombiana de Investigación Agropecuaria. Villavicencio, Colombia: Corpoica, 2014. 60 p. ISBN: 978-958-740-190-5. Recuperado el 24 de febrero de 2021 de https://repository.agrosavia.co/bitstream/handle/20.500.12324/1068/74233 65660.pdf?sequence $\% 20$ $=1 \&$ isAllowed $=\mathrm{y}$

Bolívar, H. (2011). Metodologías e indicadores de evaluación de sistemas agrícolas hacia el desarrollo sostenible. CICAG: Revista del Centro de Investigación de Ciencias Administrativas y Gerenciales, ISSN-e 1856-6189, 8(1), 1-18.

Bustamante, C. \& Rojas-Salazar, L. (2018). Reflexiones sobre transiciones ganaderas bovinas en Colombia, desafíos y oportunidades. Biodiversidad en la Práctica, 3(1), 1-29.

Cabrales R., Montoya R. \& Rivera J. (2007). Evaluación agronómica de genotipos de sorgo y millo (Sorghum vulgare) forrajeros para alimentación de bovinos en el medio sinú. Revista Medicina Veterinaria y Zootecnia Córdoba, 12(2), 1036-1041.

Carrasco, N., Zamora, M. \& Melín, A. (2011). Manual de Sorgo. 1st ed. Argentina. Instituto Nacional de Tecnologías Agropecuarias INTA. ISBN: 978-987-679-071-0. Recuperado el 22 de febrero de 2021 de https://inta.gob.ar/sites/default/files/inta_manual_de_sorgo_renglon_191.pdf

Centro Internacional de Agricultura Tropical - CIAT. (1990). Sorgo para suelos ácidos. Memorias de un taller sobre la evaluaci6n del sorgo por su tolerancia a los suelos tropicales de América Latina que contienen aluminio tóxico, Cali, Colombia, mayo 28 a junio 2 de 1984. Salinas, J. G. y Oourley, L M. (ed.). Cali, Colombia. 354 p.

Chao-Chen, T., Xiao-Lin, Y. \& Guang-Hui, X. (2018). Establishing sustainable sweet sorghum- based cropping systems for forage $\mathrm{T}$ and bioenergy feedstock in North China Plain. Field Crops Research, 227 (2018), 144-154. https://doi.org/10.1016/j.fcr.2018.08.011

Corral-Luna, A., Domínguez-Díaz, D., Rodríguez-Almeida, F., Villalobos-Villalobos, G., Ortega- Gutiérrez, J. \& Muro-Reyes, A. (2011). Composición química y cinética de degradabilidad de ensilaje de maíz convencional y sorgo de nervadura café. Revista Brasileira de Ciencias Agrarias, 6 (1), 181-187.

Contexto Ganadero. (2018, junio 28). El sorgo es otra alternativa de alimento en ensilaje. Ganadería sostenible. Contexto Ganadero. Una Lectura rural de la realidad colombiana. Recuperado el 22 de febrero de 2021 de https://www.contextoganadero.com/ganaderia-sostenible/jn-27-el-sorgo-es-otra-alternativa-de-alimento-en-ensilaje

De León, M. (2007). El uso de silajes de sorgo en la intensificación de los sistemas de producción de carne bovina. Informe Técnico $\mathrm{N}^{\mathrm{o}} 5$. Proyecto ganadero Regional. Instituto Nacional de Tecnologías Agropecuarias - INTA. Recuperado el 22 de febrero de 2021 de http://www.produccion-animal. com.ar/produccion_y_manejo_reservas/reservas_silos/76-uso_silajes_en_bovinos.pdf

De León, M. (2014, septiembre 30). La utilización de silajes en los sistemas ganaderos. Instituto Nacional de Tecnologías Agropecuarias - INTA. Recuperado el 22 de febrero de 2021 de https://inta.gob.ar/documentos/la-utilizacion-de-silajes-en-los-sistemas-ganaderos-0 
Rios Moyano, D. K.; Conde Pulgarín, A.; Rios Moyano, C. F.

De León, M. (2019, mayo 31). El sorgo gana protagonismo en las dietas ganaderas. Instituto Nacional de Tecnologías Agropecuarias - INTA. Recuperado el 22 de febrero de 2021 de https://inta.gob.ar/documentos/el-sorgo-gana-protagonismo-en-las-dietas-ganaderas

Devine, G. J., Eza, D., Ogusuku, E. \& Furlong, M. J. (2008). Uso de insecticidas: contexto y consecuencias ecológicas. Revista Peruana de Medicina Experimental y Salud Publica, 25(1), 74-100.

Díaz, M. G., López, R., Kuttel, W. \& Bendersky, D. (2011). Caracterización e identificación de germoplasmas de sorgo con aptitud silera en Paraná (Entre Ríos) y Mercedes (Corrientes). Campaña 2009/2010. INTA. Recuperado el 20 de febrero de 2021 de https://inta.gob.ar/sites/default/files/ script-tmp-inta-caracterizacion-e-identificacion-de-germoplasmas_1.pdf

Diez, M. \& Sardiña, C. (2012). Ensayo comparativo de materiales de sorgo para silo en ambientes con y sin limitantes edáficas. Recuperado el 22 de febrero de 2021 de https://inta.gob.ar/sites/default/ files/script-tmp-inta_mt2012 diez_ensayo_comparativo_materiales.pdf

Fassio, A., Cazzolino, D., Ibañez, W. \& Fernández, E. (2002). Sorgo: destino forrajero. ISBN: Editado por la Unidad de Agronegocios y Difusión del INIA. ISBN: 9974-38-160-6. Recuperado el 20 de febrero de $2021 \mathrm{de} \mathrm{http://www.inia.uy/Publicaciones/Documentos \% 20compartidos/111219240807141139.pdf}$

Federación Colombiana de Ganaderos. (2013). Programa Nacional de alimentación Bovina - PAB. Recuperado el 20 de febrero de $2021 \mathrm{de}$ https://www.slideshare.net/Fedegan/documento-pab

Federación Nacional de Cultivadores de Cereales y Leguminosas. (2010). El cultivo del sorgo, historia e importancia. Recuperado el 19 de febrero de 2021 de https://repository.agrosavia.co/bitstream/ handle/20.500.12324/19500/45187 61385.pdf?sequence=1\&isAllowed=y

Fideicomisos Instituidos en Relación con la Agricultura. (2019). Panorama Agroalimentario Sorgo 2019. Dirección de Investigación y Evaluación Económica Sectorial. Recuperado el 21 de febrero de 2021 de: https:// www.inforural.com.mx/wp-content/uploads/2019/06/Panorama-Agroalimentario-Sorgo-2019.pdf

Flores, F. (2010). Manejo de Plagas en el cultivo de maíz. Estación experimental agropecuaria Marcos Juarez. Instituto Nacional de Tecnologías Agropecuarias - INTA. Recuperado el 22 de febrero de 2021 de https://inta.gob.ar/sites/default/files/script-tmp-inta-manejo_de plagas_en_el_cultivo_de_maz.pdf

Garcés Molina, A., Berrio Roa, L., Ruíz Alzate, S., Serna DLeón, J. \& Builes Arango, A. (2004). Ensilaje como fuente de alimentación para el ganado. Revista Lasallista de Investigación, 1 (1), 66-71.

Giménez, R., Despósito, C., Burghi, V. \& De León, M. (2018). Sorgo para la confección de silajes: Su utilización en los sistemas ganaderos del noroeste de Córdoba. Recuperado el 18 de febrero de $2021 \mathrm{de}$ https://inta.gob.ar/sites/default/files/inta_sorgo_para la confeccion_de silajes_su_utilizacion_en_los_sistemas_octubre 12 2018.pdf

Gutiérrez, F., Estrella, A., Irazábal, E., Quimiz, V., Portilla, A. \& Bonifaz, N. (2018). Mejoramiento de la eficiencia de la proteína de los pastos en bovinos de leche utilizando cuatro formulaciones de balanceados. LA GRANJA. Revista de Ciencias de la Vida, 28(2), 115-122. http://doi.org/10.17163/lgr.n28.2018.09 
Productividad y sostenibilidad del cultivo de sorgo forrajero como alternativa para la alimentación de rumiantes

Iqbal, M. A., Ahmad, B., Shah, M. H. \& Ali, K. (2015). A Study on Forage Sorghum (Sorghum bicolor L.) Production in Perspectives of White Revolution in Punjab, Pakistan: Issues and Future Options. American-Eurasian Journal of Agriculture and Environmental Sciences (JAES), 15 (4), 640-647. https://www.idosi.org/aejaes/jaes 15(4)15/24.pdf

Janssen, R., Rutz, D., Braconnier, S., Reddy, B., Rao, S., Schaffert, R., Parella, R., Zaccharias, A., Rettenmaier, N., Reinhardt, G., Monti, A., Amaducci, S., Marocco, A., Snijman, W., Terblanche, H. \& Zavala-Garcia, F. (2015). Sweet sorghum - an alternative energy crop. Recuperado el 21 de febrero de 2021 de https://www. researchgate.net/publication/229013045 SWEET_SORGHUM-AN_ALTERNATIVE_ENERGY CROP

Kent, F. (2019). Ensilaje de sorgo y maíz. Instituto Nacional de Tecnologías Agropecuarias - INTA. Recuperado el 20 de febrero de $2021 \mathrm{de}$ https:/inta.gob.ar/sites/default/files/inta_federico_kent_ensilajes_sra_realico.pdf

La República. (2019 16 de abril). "La inversión en investigación está por debajo de 0,5\% del PIB en la región”: Alicia Bárcena. Recuperado el 18 de febrero de 2021 de https:/www.larepublica.co/globoeconomia/lainversion-en-investigacion-esta-por-debajo-de-05-del-pib-en-la-region-alicia-barcena-2852018

Ledbetter, K. (2019). El ensilaje de sorgo es una alternativa adecuada al ensilaje de maíz, con un manejo adecuado. Universidad de Texas A\&M. Recuperado el 22 de febrero de 2021 de https:/mundoagropecuario.com/ el-ensilaje-de-sorgo-es-una-alternativa-adecuada-al-ensilaje-de-maiz-con-un-manejo-adecuado/ .

Mahecha, L., Gallego, L. A. \& Peláez, F. J. (2002). Situación actual de la ganadería de carne en Colombia y alternativas para impulsar su competitividad y sostenibilidad. Revista Colombiana de Ciencias Pecuarias, 15 (2), 213-225.

Milesi, O. (2016). Ganadería, oportunidad y amenaza para una América Latina sostenible. Sitio Argentino de Producción Animal. Recuperado el 19 de febrero de 2021 de http://www.produccion-animal.com.ar/ informacion tecnica/origenes evolucion_y estadisticas de la ganaderia/183-ganaderia america.pdf

Ministerio de Agricultura y Desarrollo - MinAgricultura. (2014, septiembre 10). Corpoica Lanza la Primera Variedad de Sorgo Dulce Forrajero en Colombia de Gran Adaptación en Épocas de Sequía. Agronet. Red de Información y Comunicación del Sector Agropecuario Colombiano. Recuperado el 18 de febrero de 2021 de https://www.agronet.gov.co/Noticias/Paginas/Noticia1084.aspx

Ministerio de Agricultura y Desarrollo Rural - MinAgricultura. (2018). Ganaderos tienen en la siembra de sorgo forrajero una buena alternativa de alimentación para sus animales. Recuperado el 20 de febrero de 2021 de https:/www.minagricultura.gov.co/noticias/Paginas/Ganaderos-tienen-en-la-siembra-de-sorgo- forrajero-una-buena-alternativa-de-alimentaci\%C3\%B3n-para-sus-animales--.aspx

Mora Marín, M. A., Ríos Pescador, L., Ríos Ramos, L. \& Almario Charry, J. L. (2017). Impacto de la actividad ganadera sobre el suelo en Colombia. Revista Ingeniería y Región, 17(1), 1-12. https://doi.org/10.25054/22161325.1212

Morell, A., Exposito, A. M. \& Ruiz, M. (2018). El Sorgo. Una Alternativa económica y sostenible de alimento en el municipio Jobabo. Revista Desarrrollo Local Sostenible, 11(31). 
Rios Moyano, D. K.; Conde Pulgarín, A.; Rios Moyano, C. F.

Murgueitio, E. \& Ibrahim, M. (2004). Ganadería y medio ambiente en América Latina. Recuperado el 17 de febrero de $2021 \mathrm{de}$ https://www.researchgate.net/publication/237495139 Ganaderia_y medio ambiente en_America_Latina

Oliver, A. L., Grant, R. J., Pedersen, J. F. \& O’Rear, J. (2004). Comparison of brown midrib-6 and -18 forage sorghum with conventional sorghum and corn silage in diets of lactating dairy cows. Journal of Dairy Science, 87 (3), 637-644.

Organización de las Naciones Unidas para la Alimentación y la Agricultura. (2005). Alternativas nutricionales para la época seca. Recuperado el 21 de febrero de 2021 de http://www.fao.org/3/a-at783s.pdf

Organización de las Naciones Unidas para la Alimentación y la Agricultura. (2016). Desarrollo agrícola sostenible para la seguridad alimentaria y la nutrición: ¿qué función desempeña la ganadería? Recuperado el 21 de febrero de 2021de http://www.fao.org/3/a-i5795s.pdf

Organización de las Naciones Unidas para la Alimentación y la Agricultura. (2018). Transformar la alimentación y la agricultura para alcanzar los ODS, 20 acciones interconectadas para guiar a los encargados de adoptar decisiones. Recuperado el 21 de febrero de 2021 de http://www.fao.org/3/i9900es/19900ES.PDF

Organización de las Naciones Unidas para la Alimentación y la Agricultura. (2020a). El papel de la FAO en la producción animal. Producción animal. Recuperado el 21 de febrero de $2021 \mathrm{de}$ http://www.fao.org/animal-production/es/

Organización de las Naciones Unidas para la Alimentación y la Agricultura. (2020b) Ganadería sostenible y cambio climático en América Latina y el Caribe. Recuperado el 21 de febrero de 2021 de http://www.fao.org/americas/prioridades/ganaderia-sostenible/es/

Pérez, A., Saucedo, O., Iglesias, J., Wencomo, H. B., Reyes, F., Oquendo, G. \& Milián, Idolkys. (2010). Caracterización y potencialidades del grano de sorgo (Sorghum bicolor L. Moench). Estación Experimental de Pastos y Forrajes "Indio Hatuey" Matanzas, Cuba. Pastos y Forrajes, 33 (1), enero-marzo, 1-26.

Pezo, D. (2019). Intensificación sostenible de los sistemas ganaderos frente al cambio climático en América Latina y el Caribe: estado del arte. Monografía del BID. Recuperado el 20 de febrero de $2021 \mathrm{de} \mathrm{https://publications.iadb.org/es/intensificacion-sostenible-de-los-sistemas-ganade-}$ ros-frente-al-cambio-climatico-en-america-latina

Pino, F. \& Heinrichs, A. J. (2017). Sorghum forage in precision-fed dairy heifer diets. American Dairy Science Association. Journal of Dairy Science. 100, 224-235. https://doi.org/10.3168/jds.2016-11551

Resch M., Bande-Castro M. J., Pereira-Crespo S., Fernández-Lorenzo B. \& Flores G. (2013). Evaluación de variedades comerciales de sorgo forrajero en siembras tardías en la galicia atlántica: i. rendimiento en materia seca. Instituto Galego de Calidade Alimentaria. Centro de Investigacións Agrarias de Mabegondo (INGACAL-CIAM). Recuperado el 17 de febrero de 2021 de http://ciam.gal/uploads/publicacions/727archivo.pdf 
Productividad y sostenibilidad del cultivo de sorgo forrajero como alternativa para la alimentación de rumiantes

Ribeiro, L., Rodríguez, N. \& Gonçalves, L. (2007). Consideraciones sobre ensilajes de sorgo. Jornada sobre Producción y Utilización de Ensilajes-Octubre de 2007. Recuperado el 20 de febrero de 2021 de http://www.cpatsa.embrapa.br/public eletronica/downloads/OPB1703.pdf

Rocamundi, A. (2007). Silaje de sorgo "Aspectos cuantitativos del material genético existente en sorgo, con destino a silaje de planta entera”. Instituto Nacional de Tecnologías Agropecuarias - INTA. Recuperado el 17 de febrero de 2021 de http://agro.unc.edu.ar/ nutri/pdf/Sorgos\%20III.pdf

Romero, E. (2019). Los sorgos bioenergéticos. Estación experimental agroindustrial Obispo Colombres. Tucumán, Argentina. Recuperado el 18 de febrero de 2021 de http:/www.fao.org/3/ca3344es/ca3344es.pdf

Rojas, C. (2020). Informe de Estudios Económicos 2020. Cámara de Comercio de Casanare. Yopal, Casanare, Colombia. Recuperado el 20 de febrero de 2021 de https://www.cccasanare.co/wp-content/ uploads/2020/03/Estudios-económicos-vigencia-2019-1.pdf

Ruz-Reyes R., Escalona-Peña A. \& Romero-Arias A. (2018). Evaluación de tres cultivares de sorgo [Sorghum bicolor (L.) Moench] para la alimentación animal. Pastos y Forrajes, 41 (2), April-June, 86-89.

Saeed, I. \& El-Nadi, A. (1998). Forage sorghum yield and water use efficiency under variable irrigation. Irrigation Science, 18, 67-71. https://doi.org/10.1007/s002710050046

Santos, S. (2015, 12 de febrero). El ensilaje es la nueva tecnología a implementar en la ganadería. Contexto GANADERO. Una Lectura rural de la realidad colombiana. Recuperado el 17 de febrero de 2021 de https://www.contextoganadero.com/reportaje/el-ensilaje-es-la-nueva-tecnologia-implementar-en-la-ganaderia

Schuschny, A. \& Soto, H. (2009). Guía metodológica: Diseño de indicadores compuestos de desarrollo sostenible. Comisión Económica para América Latina y el Caribe (CEPAL). Impreso en Naciones Unidas, Santiago de Chile. Recuperado el 21 de febrero de 2021 de https://repositorio.cepal.org/ bitstream/handle/11362/3661/S2009230_es.pdf?sequence $=1 \&$ isAllowed $=\mathrm{y}$

Serra J., Capellades G., Salvia J. \& Aragay M. (2008). Adaptación y valor nutritivo de variedades de sorgo forrajero (Sorghum bicolor (1.) moensch) 'brown midrib' con diferente sensibilidad al fotoperiodo, en el nordeste de cataluña. Pastos, XXXVIII (1), 65 - 73

Servicio Nacional de Aprendizaje. (2009). Proyectos Pecuarios, Evidencias de las Prácticas Pecuarias Banco de Proteína - Proyecto Sena para la Implementación de un Banco de Proteína. Recuperado el 19 de febrero de $2021 \mathrm{de}$ http://produccionpecuariasena2008.blogspot.com/2009/02/banco-de- proteina.html

Torecillas, M. (2006). Sorgo para silo. Ganadería. Recuperado el 20 de febrero de 2021 de https://www.engormix.com/ganaderia-carne/articulos/sorgo-silo-t26722.htm

Tropical Cis. (2014). Sorgo para Silaje. Sorgo 600D. Recuperado el 17 de febrero de 2021 de https://tropicalcis.com/producto/sorgo-silero/

Vargas, C. F. (2005). Valoración nutricional y degradabilidad ruminal de genotipos de sorgo forrajero (Sorghum sp). Agronomía mesoamericana, 16(2), 215-223. 
Varona, M., Castro, R. A., Perez, M. I., Carvajal, N., Barbosa, E., León, L. M. \& Díaz, S. M. (2012). Impacto en la salud y el medio ambiente por exposición a plaguicidas e implementación de buenas prácticas agrícolas en el cultivo de tomate, Colombia, 2011. Revista Chilena de Salud Pública, 16 (2), $96-106$.

Villegas, J. F. \& Trujillo J. M. (2014). Herramientas para mejorar la producción y calidad de los ensilajes de maíz en Colombia. Federación Colombiana de Ganaderos - FEDEGAN -. Bogotá, Colombia. Recuperado el 21 de febrero de 2021 de https://www.fedegan.org.co/herramientas-para-mejorar-la-produccion-y-calidad-de-los-ensilajes-de-maiz-en-colombia

Xie, Q. \& Xu, Z. (2019). Sustainable Agriculture: From Sweet Sorghum Planting and Ensiling to Ruminant Feeding. Molecular Plant, 12, 603-606. https://doi.org/10.1016/j.molp.2019.04.001

Zinck, J. A., Berroterán, J. L., Farshad, A., Moameni, A., Wokabi, S. \& Van Ranst, E. (2005). La sustentabilidad agrícola: un análisis jerárquico. Secretaría de Medio Ambiente y Recursos Naturales Distrito Federal, México. Gaceta Ecológica, 76, julio-septiembre, 53-72. 